\title{
The 2019 J. Willard Gibbs Phase Equilibria Award
}

The 2019 Gibbs Award was awarded to Dr. Patrice E.A. Turchi for "outstanding and pioneering contributions in the application of first-principles, quantum-mechanical calculations to the modeling of phase equilibria and thermodynamic behavior of alloys." Dr. Turchi's work is an outstanding example of excellence in materials science in terms of scholarship, leadership in technical research, and society service and I truly cannot think of anyone more deserving for this major recognition.

Throughout his distinguished career, Dr. Turchi demonstrated how quantum-mechanical methods could be used as a framework for computing the energetics of both

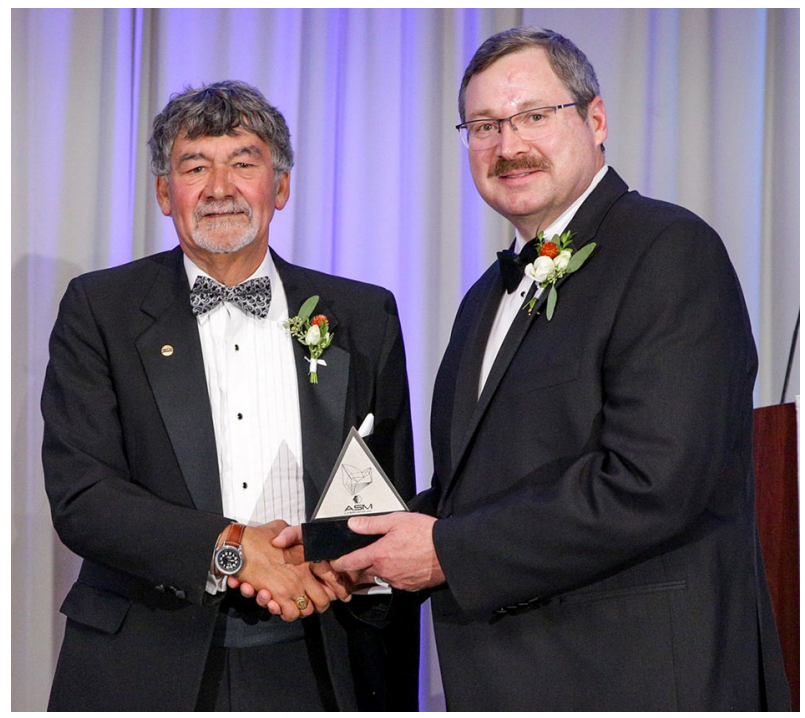

Dr. Patrice Turchi receiving the J. Willard Gibbs Phase Equilibria Award from ASM International President David Furrer ordered and disordered substitutional alloys from first principles (i.e., without data from experimental measurements or empirical models), and how this information could be combined with statistical-mechanical models for configurational entropy, to derive alloy free energies and ultimately phase diagrams. In the early 1990s, Dr. Turchi achieved the first calculation of a solid-state binary alloy phase diagram (i.e., $\mathrm{Cu}-\mathrm{Zn}$ ) entirely from a quantummechanical starting point. As Dr. Turchi's driving force is to enable practical applications of alloy theory to real engineering materials, he was also amongst the first in the field to successfully extend quantum-mechanical methods to multicomponent alloy phase stability. Within this context and the recent development of new refractory high entropy alloys, some of Dr. Turchi's alloy-theory works on

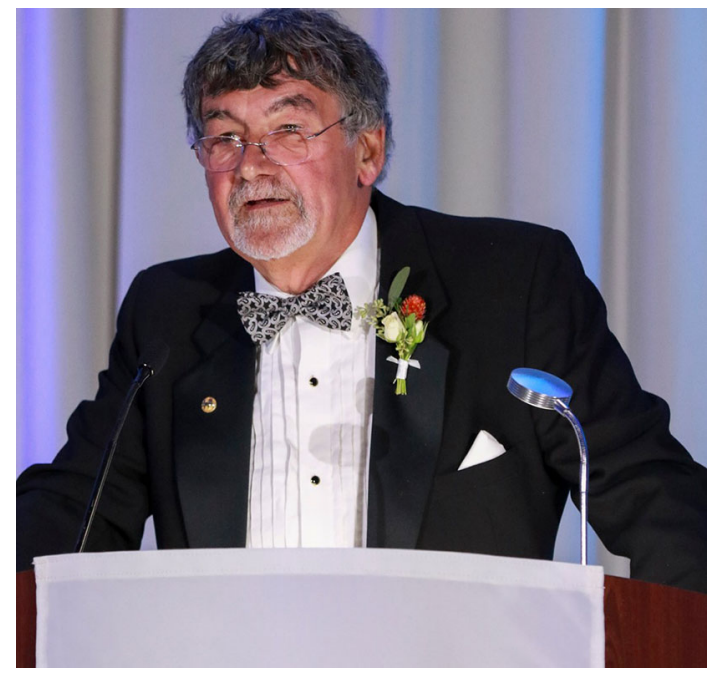

Dr. Patrice Turchi speaking at the ASM Awards Dinner 
phase separation and ordering in refractory metal alloys are presented in this special issue.

But Dr. Turchi did not stop there. Guided by his will to bring together the alloy-theory and CALPHAD (calculation of phase diagrams) communities, he made enormous contributions to breaking down barriers due to differences in fields' philosophy that were exacerbated by an inadequate understanding on both sides of the advantages and limitations of first principles and computational thermodynamic formalisms. Dr. Turchi published a series of papers demonstrating how first-principles density-functionaltheory (DFT) calculations could be integrated within the framework of CALPHAD assessments, to improve the accuracy of computational thermodynamics descriptions of multicomponent phase diagrams. The type of approach that was undertaken in those papers was practiced by very few groups at the time, and Dr. Turchi's contributions, in collaboration with his good friend the late Larry Kaufman, were influential in demonstrating how the seemingly distinct methodologies of DFT and CALPHAD could be integrated. Today, the use of DFT calculations in phase diagram assessments has become a standard mode of operation, particularly in cases where insufficient experimental data is available to enable reliable phase-diagram assessments. A showcase for where such approaches are essential has been in the application of CALPHAD methods to the modeling of actinide-alloy phase diagrams, an area where Dr. Turchi has made truly unique contributions, and is illustrated by the contribution of Perron and Turchi in this special issue. Always pushing for a better fundamental understanding of thermodynamic behavior in alloys to support their practical applications, Dr. Turchi contributed to further linking CALPHAD with microstructure evolution modeling (e.g. phase field model) and alloy design (e.g. high throughput calculations). Most recently, he provided the theoretical underpinning that was critical in the development of a new class of aluminum casting alloys based upon the Al-Ce system that is rapidly moving into commercial production. In his extensive research, he has made significant contributions to practical applications, as illustrated by the contributions of Moore and co-workers (development of new Al-Ce-based alloys), Henein and coworkers (rapid solidification of $\mathrm{Al}$ alloys), Zhang and coworkers (simulation of the solidification of casting alloys), and Xiong and co-workers (high-throughput CALPHAD calculations).
Finally, Dr. Turchi's dedication extends way beyond his scientific contribution. He is an engaged member of the scientific community, for example, from 2010 to 2013 he chaired the Alloy Phase Diagram Committee of ASM. His commitment to promoting the dissemination of the latest developments in his field through the organization of conferences and workshops, mentoring, and professional society involvement has been of enormous benefit to our community. I have been extremely lucky to have Dr. Patrice Turchi as a mentor, and I wish we could all one day have his knowledge, enthusiasm, sense of responsibilities and humor, and leadership.

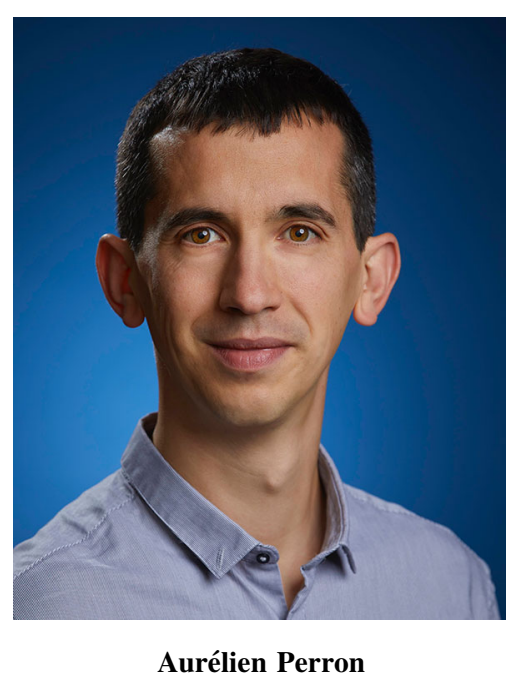

\author{
Aurélien Perron \\ Deputy Leader, Actinide and Lanthanide Science Group \\ Materials Science Division \\ Lawrence Livermore National Laboratory \\ Contact e-mail: perron1@llnl.gov
}

This editorial represents my professional opinion and is not an official Lawrence Livermore National Security, LLC (LLNS) endorsement of the individual. Prepared by LLNL under Contract DE-AC52-07NA27344.

Publisher's Note Springer Nature remains neutral with regard to jurisdictional claims in published maps and institutional affiliations. 